\title{
Insulinoma en una paciente con infección por VIH en tratamiento antirretroviral
}

\section{Insulinoma in a patient with HIV infection on antiretroviral treatment}

\author{
José Luis Paz-lbarra',2 \\ https://orcid.org/0000-0002-285I-3727
}

\section{Karla Carolina Salas-Rodríguez'}

https://orcid.org/0000-0002-5049-74IX

Víctor Raúl García-Ruiz'

https://orcid.org/0000-0002-6846-7630
Paz-lbarra J, Salas-Rodríguez K, García-RuizV. Insulinoma en una paciente con infección por VIH en tratamiento antirretroviral. Rev Soc Peru Med Interna. 2020;33(4): I64-I67.

https://doi.org/10.36393/spmi.v33i4.565

\section{RESUMEN}

Se presenta el caso de una paciente de 43 años con el diagnóstico de infección por VIH en tratamiento antiviral que desarrolló convulsiones sin respuesta al tratamiento anticonvulsivante. El estudio metabólico y estructural evidenció la presencia de hipoglicemia hiperinsulinémica cuya evaluación concluyó que ella tenía un insulinoma por lo que fue sometida a pancreatectomía subtotal sin recurrencia de episodios convulsivos en el seguimiento.

Palabras Clave:VIH, antirretroviral, convulsiones, insulinoma, hipoglucemia (DeCS BIREME).

\section{ABSTRACT}

We present the case of a 43-year-old patient with a diagnosis of HIV infection on antiviral treatment who developed seizures without response to anticonvulsant treatment. The metabolic and structural study showed the presence of hyperinsulinemic hypoglycemia whose evaluation concluded that she had an insulinoma and was therefore subjected to subtotal pancreatectomy without recurrence of seizure episodes during the follow-up.

Keywords: HIV, antiretroviral, seizures, insulinoma, hypoglycemia (MeSH NLM)

I. Médico endocrinólgo. Servicio de Endocrinología, Hospital Nacional EsSalud Edgardo Rebagliati Martins. Lima, Perú.

2. Departamento de Medicina, Universidad Nacional Mayor de San Marcos. Lima, Perú.

\section{INTRODUCCIÓN}

El insulinoma es el tumor funcionante de los islotes pancreáticos más frecuente, con una incidencia de 1-4 casos/millón de personas/año. ${ }^{1}$ Whipple definió la manifestación clásica del insulinoma con su famosa tríada que incluye hipoglicemia (glicemia $<40 \mathrm{mg}$ / dl), síntomas neuroglicopénicos y alivio rápido de los síntomas tras la administración de glucosa. ${ }^{2}$ Esta triada puede estar presente en otras patologías como la nesidioblastosis, el ayuno, causas autoinmunes, deficiencia de hormonas contrarreguladoras, inducida por fármacos. ${ }^{3}$

En el contexto de la infección por VIH se describen eventos autoinmunes en $11-72 \%$ de los pacientes, pero en su mayoría no presentan manifestaciones clínicas sistémicas, con mayor frecuencia en estadios de la infección cuando los linfocitos CD4+ son normales (estadio precoz o por recuperación inmunológica tras la terapia antirretroviral. Estos mecanismos 
autoinmunes se plantean por el mimetismo molecular, invasión directa por el virus, activación policlonal de las células $\mathrm{B}$, factores genéticos y ambientales; sin embargo, aún no está descrito la presencia de anticuerpos antiinsulina. . $^{3,4}$

La terapia antirretroviral (TAR) de gran actividad ha cambiado la historia natural de la infección por $\mathrm{VIH}^{5}$, surgiendo así manifestaciones endocrinas relacionadas a una mayor esperanza de vida obtenida con el desarrollo de condiciones metabólicas que afectan la calidad de vida..$^{3,6}$

Presentamos el caso de una paciente con VIH y en tratamiento antirretroviral con un insulinoma que debuta con síndrome convulsivo asociado a hipoglicemia y que se resuelve tras el diagnóstico y tratamiento quirúrgico oportuno.

\section{PRESENTACIÓN DEL CASO}

Paciente mujer de 43 años de edad con infección por VIH desde hace siete años y con tratamiento de lamivudina, tenofovir, atazanavir. Tres años antes de su ingreso empezó a presentar palpitaciones, sudoración, somnolencia, de predominio matutino, y que los síntomas mejoraban con la ingestión de alimentos dulces. Hace dos años después presentó, mientras dormía, un episodio convulsivo que duró dos minutos, cediendo espontáneamente y en el estado postictal mejoró con la ingestión de alimentos dulces. Al presentar otros episodios similares, con una RMN normal, en neurología, se le inició tratamiento con fenitoína y se agregó, después, fenobarbital por persistencia de las convulsiones; y, observó que con el aumento de la cantidad y frecuencia de alimentos disminuyeron los episodios convulsivos. Un año antes del ingreso, en consulta ambulatoria de infectología, presentó glicemia en ayunas de $46 \mathrm{mg}$ / dl y fue referida a endocrinología para estudio.

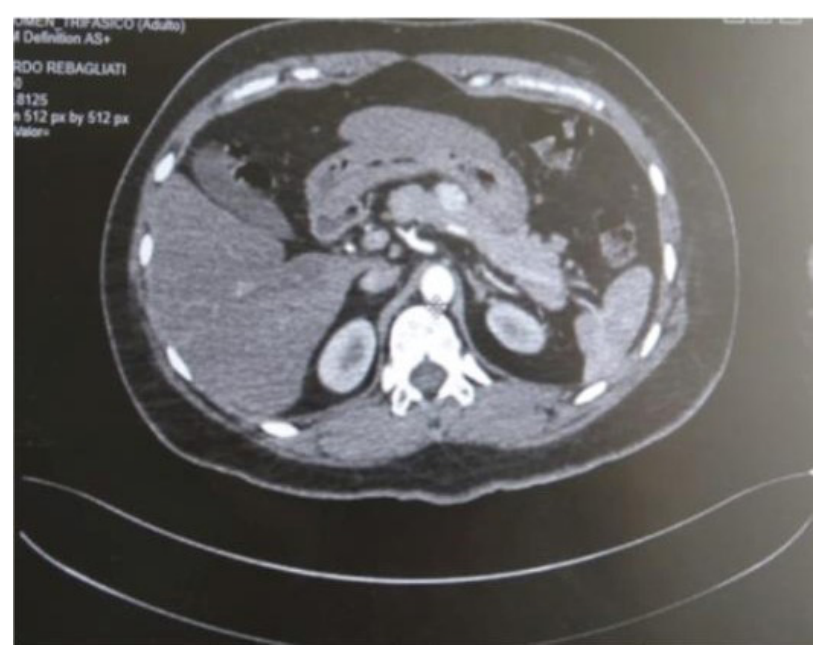

Figura I. TC dinámica: lesión hipervascularizada en el cuerpo del páncreas en la fase arterial precoz.
En hospitalización: Peso 65,5kg, IMC 28,36 kg/m²; $\mathrm{y}$, el resto del examen físico fue normal. Presentó glicemia capilar en ayunas de 22 y $43 \mathrm{mg} / \mathrm{dl}$ en dos días sucesivos con síntomas autonómicos.

Los exámenes auxiliares: TSH 2,9u U/ml (VN: 0,44,0), T4L 0,952ng/dl (VN: 0.8-1.9); cortisol (8am) 14,7ug/dl (VN: 5-25); IGF1120ng/ml (VN:117-329); creatinina $0,62 \mathrm{mg} / \mathrm{dl}$, pruebas de función hepática normales; y, carga viral: indetectable. Fue sometida a prueba de ayuno la cual se terminó a las 6 horas al presentar síntomas asociados a glicemia capilar $<45 \mathrm{mg}$ / dl, glicemia plasmática $38 \mathrm{mg} / \mathrm{dl}$, insulinemia $25,2 \mathrm{uUI} / \mathrm{ml}$, péptido C $4,2 \mathrm{ng} / \mathrm{ml}$; anticuerpos antiinsulina (-). RMN de encéfalo: normal. TC pancreática trifásica mostró un nódulo de 13 x8 mm en el borde anterior del cuerpo del páncreas, sugestivo de tumor neuroendocrino (TNE) en el estudio dinámico (Figura 1); y, la ecoendoscopía mostró una lesión hipoecogénica de 13,4 x 15,0 mm, de bordes bien definidos en el cuerpo proximal y anterior de páncreas (Figura 2).

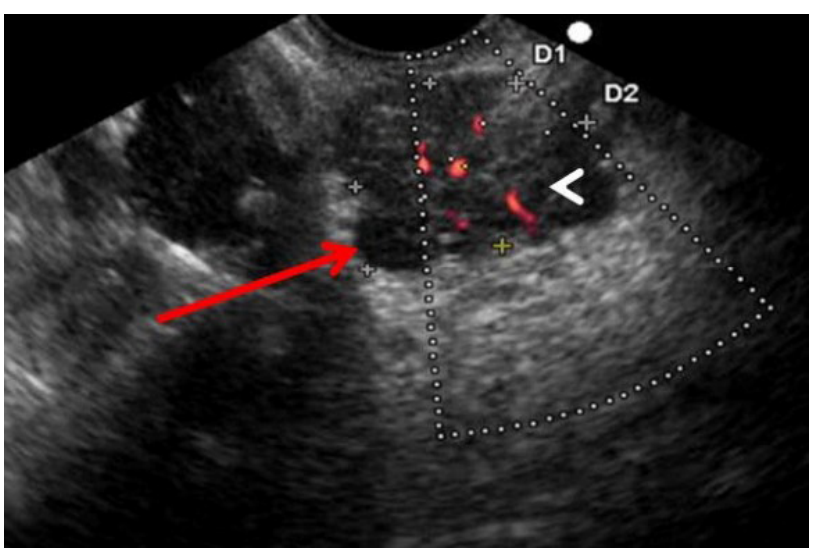

Figura 2. Eco-endoscopia: lesión hipoecogénica en el cuerpo del páncreas.

Al no ser posible la enucleación del tumor, se realizó pancreatectomía distal, y el cirujano reportó un tumor de $2 \mathrm{~cm}$ de consistencia aumentada, indurada y bordes delimitados. El estudio histopatológico concluyó: TNE tipo I de patrón trabecular, con márgenes quirúrgicos libres de lesión Ki67 de 4\%. Desde la cirugía y hasta la actualidad la paciente no ha presentado episodios de hipoglicemia ni convulsiones.

\section{DISCUSIÓN}

Los TNEse caracterizan por su capacidad de almacenar y secretar diferentes péptidos y neuroaminas. ${ }^{7,8}$ Más del $90 \%$ de los insulinomas son benignos, solitarios e hipervascularizados. ${ }^{1,7}$ En el enfoque diagnóstico del insulinoma, se recomienda la prueba de ayuno de 72 horas, en la que se mide continuamente las concentraciones de glicemia, insulina y péptido $\mathrm{C}$. Tiene un alto rendimiento diagnóstico en alrededor 
del $90 \%$ al $95 \%$ de los casos sometidos a un ayuno supervisado. ${ }^{7-10}$ Los estudios de imagen detectan más del $70 \%$ de los TNE pancreáticos mayores de $3 \mathrm{~cm} \mathrm{y}$ menos del $50 \%$ de los TNE pancreáticos menores a $1 \mathrm{~cm}$. La TC contrastada se ha utilizado con mayor frecuencia como el primer método diagnóstico. La ecografía endoscópica es útil en insulinomas pequeños, casi siempre intrapancreáticos, detectando el TNE en un $90 \%$ de los casos, con una sensibilidad diagnostica del $95 \%$ al $100 \%$ en la localización de lesiones intrapancreáticas. ${ }^{10}$ Por este motivo, algunos centros lo utilizan como la principal modalidad de localización preoperatoria. $^{2}$ La localización preoperatoria del insulinoma previene la pancreatectomía ciega distal y evita la reoperación, además de la determinación de la malignidad, multiplicidad, metástasis, casos complejos y la proximidad del tumor a los vasos principales. Los síntomas de hipoglicemia tanto autonómicos como neuroglicopénicos (convulsiones), acompañados por la clásica "tríada de Whipple", durante el ayuno con la exclusión de otras causas de hipoglicemia hiperinsulinémica nos permitieron plantear el diagnóstico de insulinoma. Peltola y col. describieron en una cohorte de 79 pacientes con insulinoma, que los síntomas de presentación más frecuente fueron confusión $(86 \%)$, somnolencia $(58 \%)$ y sudoración excesiva $(57 \%)$, como en nuestra paciente. $^{1}$

El uso de TAR se viene asociando al desarrollo de alteraciones metabólicas que afectan la calidad de vida de los pacientes; así, los análogos de nucleósidos inhibidores de transcriptasa reversa se han asociado con resistencia a la insulina y diabetes mellitus tipo 2 al producir una disminución del 25\% en la eliminación de la glucosa mediada por insulina y un aumento del $22 \%$ en la lipólisis en ayunas, evidenciada con el uso de zidovudina/lamivudina. ${ }^{6}$ Los inhibidores de la proteasa pueden inhibir la conversión de proinsulina a insulina, la proteasa que regula el catabolismo de la insulina, con un estado de hiperinsulinemia e hiperglucemia y / o secreción de proinsulina inmadura en las células beta. ${ }^{10,11}$ Sin embargo, en una cohorte de 30 sujetos sanos VIH negativos, el azatanavir no afectó la sensibilidad a la insulina mientras que la combinación lopinavir/ritonavir indujo la resistencia a la insulina. ${ }^{12}$ En nuestra paciente el uso de TAR puede explicar una demora en el diagnóstico, como ha sido descrito. ${ }^{6,11}$

La tasa de curación inmediata después de la resección es del $100 \%$, con tasas libres de enfermedad a 10 y 15 años de 95,6 y $85,4 \%$, respectivamente, que ha sido descrito por Tsang y col. en su cohorte de 36 pacientes operados de insulinoma, cuyo resultado concuerda con nuestra paciente. ${ }^{13}$

En conclusión, el manejo del insulinoma incluye el diagnóstico, la localización del tumor y el tratamiento en centros especializados. La sospecha de hipoglicemia hiperinsulinémica en pacientes con hipoglicemia nos permitirá un diagnóstico temprano y un adecuado manejo.

\section{REFERENCIAS BIBLIOGRÁFICAS}

1. Peltola E, Hannula P, Huhtala H. Characteristics and Outcomes of 79 Patients with an Insulinoma: A Nationwide Retrospective Study in Finland. Int J Endocrinol. 2018; 2018:2059481. Published 2018 Oct 23. doi:10.1155/2018/2059481

2. Mehrabi A, Fischer L, Hafezi M. A systematic review of localization, surgical treatment options, and outcome of insulinoma. Pancreas. 2014; 43(5):675-686.

3. Mirza FS, Luthra P, Chirch L. Endocrinological aspects of HIV infection. J Endocrinol Invest. 2018; 41(8):881-899. doi:10.1007/s40618-017-0812-x.

4. Campos M. Manifestaciones reumáticas y/o autoinmunes asociadas a la infección por el virus de la inmunodeficiencia humana. Unidad de Enfermedades Autoinmunes Sistémicas. Hospital Carlos Haya, Málaga. Junio 2010; 3(2):2125. Cuadernos de Autoinmunidad. Publicación Oficial de la Sociedad Andaluza de Enfermedades Autoinmunes.

5. Fuster M. Resistencia a la insulina en pacientes infectados por VIH en Tratamiento antirretroviral. Tesis doctoral. Universidad Complutense de Madrid. Facultad de Medicina. Madrid 2010. Disponible en: https: / / eprints.ucm.es/10228/1/ T31490.pdf

6. Öberg K. Management of functional neuroendocrine tumors of the pancreas. Gland Surg. 2018 Feb;7(1):20-27. doi: 10.21037/ gs.2017.10.08

7. Cives M, Strosberg JR. Gastroenteropancreatic neuroendocrine tumors. CA Cancer J Clin. 2018;68(6):471-487. doi:10.3322/ caac. 21493

8. Vinik AI, Woltering EA, Warner RR, et al. NANETS consensus guidelines for the diagnosis of neuroendocrine tumor. Pancreas. 2010;39(6):713734. doi:10.1097/MPA.0b013e3181ebaffd

9. Uri I, Grozinsky-Glasberg S. Current treatment strategies for patients with advanced gastroenteropancreatic neuroendocrine tumors (GEP-NETs). Clin Diabetes Endocrinol. 2018 Jul 11;4:16. doi: 10.1186/s40842-018-0066-3

10. Carr A, Samaras K, Thorisdottir A, Kaufmann GR, Chisholm DJ, Cooper DA. Diagnosis, prediction, and natural course of HIV-1 protease-inhibitorassociated lipodystrophy, hyperlipidaemia, and diabetes mellitus: a cohort study. Lancet. 1999;353(9170):2093-2099. doi:10.1016/S01406736(98)08468-2

11. Obirikorang C, Quaye L, Osei-Yeboah J, Odame EA, Asare I. Prevalence of metabolic syndrome among HIV-infected patients in Ghana: A cross- 
sectional study. Niger Med J. 2016;57(2):86-90. doi:10.4103/0300-1652.182082

12. Noor MA, Parker RA, O'Mara E, et al. The effects of HIV protease inhibitors atazanavir and lopinavir/ritonavir on insulin sensitivity in HIVseronegative healthy adults. AIDS. 2004;18 (16):2137-2144
13. Tsang Y-P, Lang BH-H, Shek TW-H. Outcomes of benign insulinomas. ANZ J Surg. 2016; 86: 706-710. doi:10.1111/ans.12891

CORRESPONDENCIA:

losé Paz-lbarra

jlpi_09@yahoo.com

Fecha de recepción: 25-09-2020.

Fecha de aceptación: |6-|| -2020. 